\title{
P 038 WHAT NOW? HOSPITAL DOCTORS' EXPERIENCES AND PERCEPTIONS OF THE LIVERPOOL CARE PATHWAY IN TIMES OF CHANGE: A QUALITATIVE STUDY
}

Sharon Twigger, ${ }^{1}$ Sarah Yardley ${ }^{2} .{ }^{1}$ West Midlands Deanery; ${ }^{2}$ Keele University

10.1136/bmjspcare-2014-000654.79

Background Introducing 'The Liverpool Care Pathway for the Dying Patient' (LCP) to acute settings in the UK was intended to transfer hospice models of high quality end-of-life care onto hospital wards. Growing criticism and concerns, (from the public, media, politicians and healthcare workers), regarding when and how the LCP was used has culminated in an independent review recommending the LCP is phased out of use. Despite this, limited research exists exploring doctors' perceptions of the LCP.

Aims To explore lived experiences of hospital doctors using the LCP, how these experiences have influenced their practices and perceptions of the LCP and how wider application in hospitals went wrong, in order to inform future interventions seeking to improve end-of-life care.

Methods Following a systematic review of the literature on use of the LCP in hospitals prospective ethical approval was received to recruit participants. Consultants and registrars, from an acute hospital in England, were purposively selected for one-to-one in-depth interviews. The topic guide covered positive and negative experiences of the LCP, individuals' perceptions, external influences and training. Interviews were audio-recorded and transcribed verbatim for thematic analysis.

Results In this paper we present our thematic findings and an interpretative analysis of lessons to be learned from the 'LCP experience' in hospitals. We will discuss how participants created 
meaning and constructed knowledge from their real life experiences. We will consider how these experiences, and external factors, influenced decision-making with respect to the LCP and end-of-life care more generally.

Conclusions The need for high quality end-of-life care in hospitals remains, regardless of how history may judge the LCP from inception to withdrawal. Now is the time for specialist palliative care to learn the lessons of the LCP era and to work with generalists, the public, media and policy-makers to improve end-of-life care for the future. 